\title{
Calibration and Correction Factors of ISO-Rad Diodes P-Type for In-Vivo Dosimetry in External Beam Therapy with Co60
}

\author{
${ }^{1}$ Nureddin. A. S. Musa, ${ }^{2}$ Pro. Mohamed Elfadil. M \\ Sudan University of Science and Technology, College of Graduate Studies, Scientific Research, P.O. Box 407, Khartoum, Sudan. \\ DOI: 10.29322/IJSRP.11.01.2021.p10989 \\ http://dx.doi.org/10.29322/IJSRP.11.01.2021.p10989
}

\begin{abstract}
Radiation therapy has been used in the treatment of malignant diseases since its discovery. It has been found that the use of radiation in the treatment in inaccurate ways causes irreparable damage and does not achieve the desired goal of treatment by itself. It was very important to use in vivo diode dosimetry techniques for radiotherapy measurement to accurately check the dose administered and deposited by the patient to during treatment. To achieve this goal, the absorbed dose verification program was initiated by using the diode detectors in vivo dosimetry system for entrance dose during treatment. The aim of this study was to verily the response of diodes p-type (Isorad) use in radiotherapy and to establish calibration procedure for diodes and to evaluate entrance dose obtained by the treatment planning system with measured dose using four Iso-rad diode detectors conducts in this study. Calibration of the diodes was done using Cobalt-60 machine, calibration factors, correction factors were determined. This practice was performed at NSIC- Libya. Diodes were calibrated for making absorbed dose measurements. Various correction factors (linearity, SSD, field size, angle of incidence, wedge and tray) were determined for diode in vivo dosimtery system. The measurements were preformed in sold phantom in order to validate the in-vivo procedure. This investigation revealed that clinical dosimetry using diodes is simple, provides immediate results and is useful quality assurance tool for dose delivery. The results indicate that the diodes exhibit excellent linearity, dose reproducibility and minimal anisotropy; that they can be used with confidence for patient dose verification as known from the literature [1.3]. Furthermore, diodes render real time verification of the dose delivered to patients.
\end{abstract}

Index Terms- Diodes, in-vivo dosimetry, entrance dose, radiotherapy, dose verification, QA

\section{INTRODUCTION}

$\mathrm{R}$ adiation treatment accuracy is expressed as a comparison between prescribed and delivered dose. Several studies suggest $3.5 \%, 1 \mathrm{SD}$ [1-2] as the overall accuracy required and achievable in radiation treatment, based on radiobiological studies and measurements by in vivo dosimetry in clinical conditions. Semiconductor diodes as detectors for in vivo dosimetry are considered as very useful tool in clinical practice. Their main advantage over other detectors, such as diode a possibility of immediate readout and detection of errors while patient is still on a treatment couch. Moreover, diodes are known for their high sensitivity, small size, simplicity of operation and mechanical stability. However, for accurate dosimetry, diodes have to be individually characterized for conditions other than referent. In this study we present first results in implementation of in-vivo dosimetry in our department by calibration and characterization of diodes designed for use in $\mathrm{Co}^{60}$ beams. It is known from the literature that ideal diode should have small dependence, of about 1-2\%, on field size, source to skin distance (SSD) and use of beam modifying devices $[1,3]$.

\section{MATERIAL AND METHODS}

Treatment machine and dosimetry system: All diode calibrations, correction factors determination carried out using One Cobalt-60 (CIRUS, CIS BIO, French AEC SN 4248, ACCT, Activity 233.TBq) unit were used with our in vivo dosimetry system. A Cobalt-60 unit is used with energy of $1.25 \mathrm{MV}$. The in vivo dosimetry system used with diodes was IVD Model (ISORAD ${ }^{\mathrm{TM}}$ Sun Nuclear Corporation, Model p-type, USA), four diodes conducting in this study labeled as A, B, C and D). Phantom made from Perspex slabs $\mathrm{C} 5 \mathrm{H} 8 \mathrm{O} 2$ of $1.15 \mathrm{gm} / \mathrm{cc}$ density the thickness of each slab is $1 \mathrm{~cm}$, the exception is the slab in which the ionization chamber is inserted, $2 \mathrm{~cm}^{2}$ and $0.5 \mathrm{~cm}^{2}$ ) phantom with a sided window of $30 \mathrm{~cm}^{2} \times 30 \mathrm{~cm}^{2}$ x $30 \mathrm{~cm}^{2}$ size were used for this study. The dose at $D_{\max }$ on the central axis was determined by the use of a calibrated ion chamber connected to electrometer was used as a reference detector for calibration. For convenience, the phantom was generally sold. Usually the standard reference condition consisted of (gantry of $0^{\circ}$, source to surface distance (SSD) of $80 \mathrm{~cm}^{2}$, field size of $10 \mathrm{~cm}^{2} \times 10 \mathrm{~cm}^{2}$ and dose of 100 cGy at $D$ max. The Cobalt-60 unit were calibrated and checked monthly for consistency by using a calibrated chamber to determine the actual dose at $D$ max to check the dose produced over time and consider it as the device's reference dose. The diode was taped on the top flat surface of the phantom along central axis. The photon beam from the $\mathrm{C} 0{ }^{60}$ unit had a nominal energy of $1.25 \mathrm{MV}$. Cobalt-60 radiation reaches its maximum dose at $0.5 \mathrm{~cm}^{2}$ below the skin surface it was especially well-for radiation therapy of the head, neck and breast and for tumors within $5 \mathrm{~cm}$ of the skin surface in other parts of the body. 


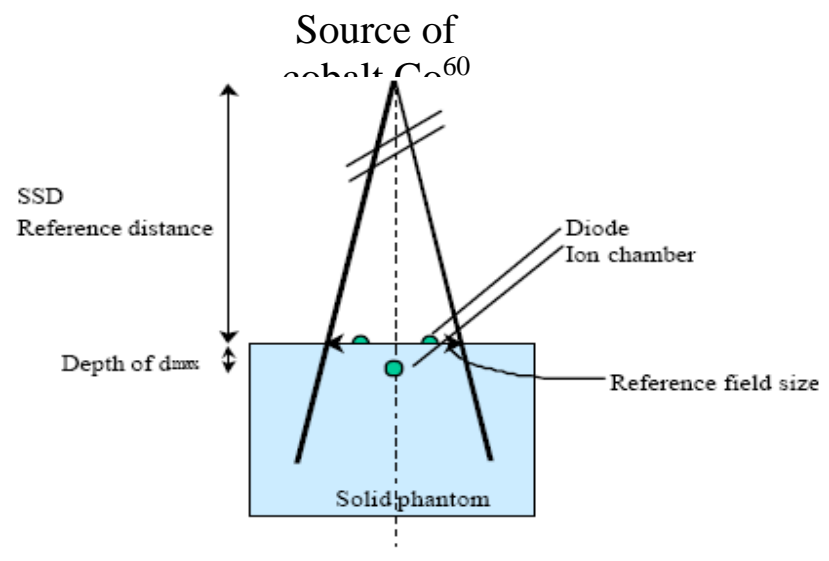

Fig.I Diode calibration procedure for dose measurements.

Calibration factors were determined for each diodes as the ratio of dose measured by ion chamber placed at $D_{\max }$ in Perspex phantom and signal from diodes placed at phantom surface, at standard reference condition, and the diode shifted slightly $2 \mathrm{~cm}^{2}$ from central axis to avoid the shadow of the entrance detector on the ionization chamber, to the detectors with respect to each other. After complete all measurements all correction factors will give true entrance dose we have simulated clinical conditions on Perspex phantom and compared diodes readings with the expected dose calculated with treatment planning system (TPS). The calibration factor is then calculated automatically using IVD software for all diode groups.

\section{CALIBRATION FACTORS}

Calibration factors procedure and determination of correction factors: calibration means the determination of calibration factors of each diode and the determination of the correction factors which are required to calculate the absorbed when measuring in clinical and calibration conditions difference. The calibration factor for entrance dose for each individual diode was determined as the ratio of the absorbed dose at dose at $d_{\max }$ to the reading of diode in reference conditions ( on the surface of the phantom at SSD of $80 \mathrm{~cm}$, with a field size of 10x10 cm and angle at zero degree $0^{\circ}$ ). After determination of the calibration factor completed, set of correction factors for field size, SSD, wedge filters and tray plate has to be established to account for changing in the diode response when measurement and calibration condition are different such as changing the distance or treatment field space. The overall factor for conversion diode reading to a measured entrance dose was obtained as the product of the dose calibration factors and the correction factors for the beam.

The manufacturer of the p-type Isorad diodes (Sun Nuclear Corporation) including other authors $[4,5]$ set detailed calibration procedures for the diodes. The calibration procedures are similar in that they use an ion chamber as a reference to obtain the required calibration factors.

In order to determine the measured dose with the diode, the following equation was used:
$\mathrm{F}_{\text {entrance }}=\frac{\text { D entrance }}{M \text { entrance }}$

Where $\mathrm{D}$ entrance is the entrance dose measured with the ion chamber at maximum dose depth and $\mathrm{M}$ entrance is the diode signal on surface. To determine the entrance calibration factor, specified in equation (1) and the diodes Sensors were irradiated under standard reference conditions setup as shown in the previous figure (1).

\section{RESULTS AND DISCUSSION}

Linearity: The diodes sensors were irradiated between $(0.5-$ $8 \mathrm{~Gy}$ ) under standard reference conditions setup.

$\frac{\text { Max. reading }}{\text { Min. reading }} \div \frac{\text { Max. time }}{\text { Min. time }}$

Linearity of each diode response to dose interval that is typical used in patient's treatment is given in figure (2), usually ranges between 0.98 and 1.02 .

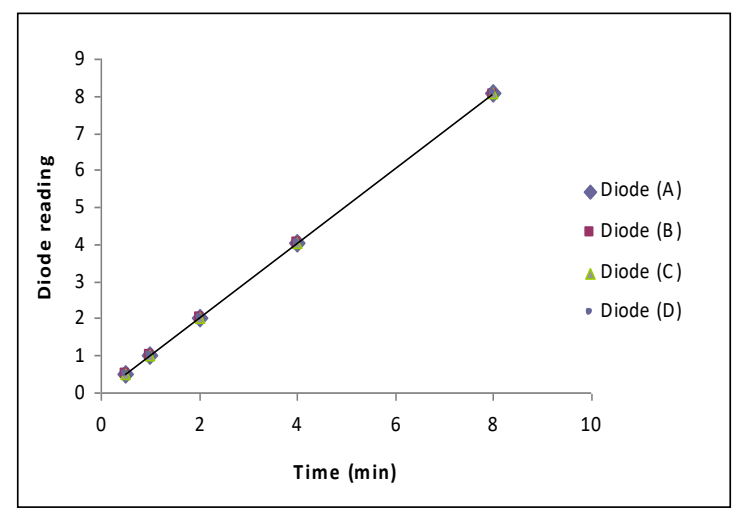

Fig II. Diodes signal as a function of the beam energy.

All diodes sensors showed acceptable intrinsic precision, less than SD recommendation of $0.5 \%$ [6]. Linearity of diodes dose response was very good in dose interval that is typical used in patient $(0.5-8 \mathrm{~Gy})$. The results for each diode together with calibration factors summarized in table (1).

Table I. Given the results for each diode together with calibration factors.

\begin{tabular}{|l|l|l|l|l|}
\hline Acceptance test & $\begin{array}{l}\text { Diode } \\
\mathrm{A}\end{array}$ & $\begin{array}{l}\text { Diode } \\
\mathrm{B}\end{array}$ & $\begin{array}{l}\text { Diode } \\
\mathrm{C}\end{array}$ & $\begin{array}{l}\text { Diode } \\
\mathrm{D}\end{array}$ \\
\hline $\begin{array}{l}\text { Intrinsic precision } \\
\text { standard deviation }\end{array}$ & $0.10 \%$ & $0.17 \%$ & $0.12 \%$ & $0.12 \%$ \\
\hline $\begin{array}{l}\text { Linearity- } \\
\text { correlation } \\
\text { coefficient stability }\end{array}$ & 1.0075 & 1.0061 & 1.0069 & 1.0051 \\
\hline $\begin{array}{l}\text { Signal ifadiation } \\
\text { after irradian } \\
\text { deviation. }\end{array}$ & 0.09 & 0.10 & 0.10 \\
\hline
\end{tabular}


Diodes were regularly recalibrated every week during one month and they showed very small sensitivity variation. That can be explained with less of accumulated dose in one week. Variation in diodes sensors sensitivity with field size was very small, producing about $0.5 \%$ overestimation of dose, for $25 \mathrm{~cm}$ square field as seen in fig .III. a. The correction factor with field size $\left(\mathrm{CF}_{\mathrm{FS}}\right)$ decreasing with increasing the field size, the reason is due to the increase in the rate of scatter in the radiation beam from the

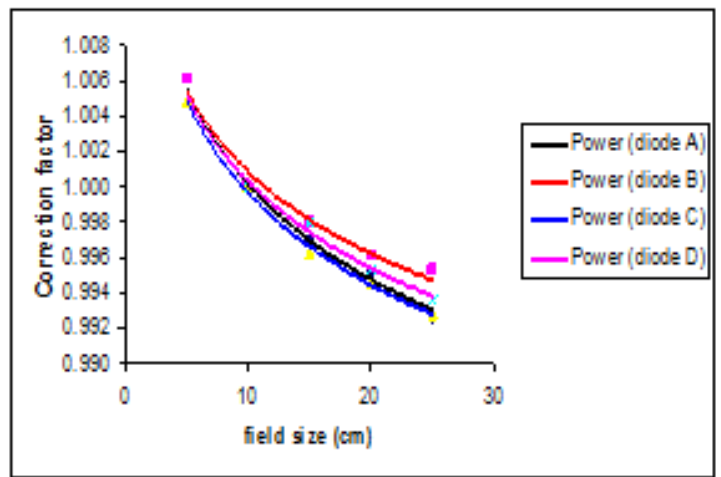

head. This is expected results for $\mathrm{Co}^{60}$ beams and EDE diodes [7, 8].

Changing the Source-surface distance (SSD) from 70 to 90 $\mathrm{cm}$ increases the $\mathrm{CF}_{\mathrm{SSD}}$ by about $4 \%$ as shown in fig. III. b. For smaller SSD there is a larger number of head scatter that reaches the diode and the ratio of the ion chamber reading is decreasing [9]. Increase in SSD results in lowering the dose rate which is another reason for underestimation of diode signal. $\mathrm{CF}_{\mathrm{SSD}}$ increasing with SSD, A, B, C and D represents four diodes used.

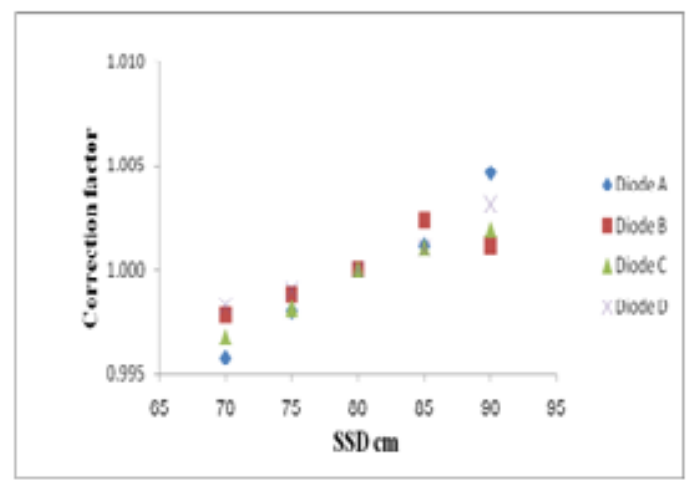

Fig .III. a. CF Fs decreasing with increasing the field size, (b) CFssD increasing with SSD, DA, DB, DC and DD represents four diodes used.

Effect of wedge filters on diode response is shown in fig. 4. a. It ranges from about $1 \%$ for small wedge $15^{\circ}, 30^{\circ}, 45^{\circ}$ and $60^{\circ}$ for all four diodes and all field size, to $6 \%$ in sensitivity variation for $60^{\circ}$ wedge. When inserting the wedge in a beam decreases the dose rate and changes the beam quality and the diodes read smaller dose than expected, the CFs are greater than one. Also when using the tray plate for supporting blocks in the beam alters the diode response by the electrons, which causing overestimation of dose for $25 \mathrm{~cm}$ square field. In this study we measured correction factors for $0.5 \mathrm{~cm}$ thick PMMA tray plate with Shielding constriction for block fixation, placed on $54.5 \mathrm{~cm}$ from the sourer. The tray plate correction factors are explained in fig. 4 . b.
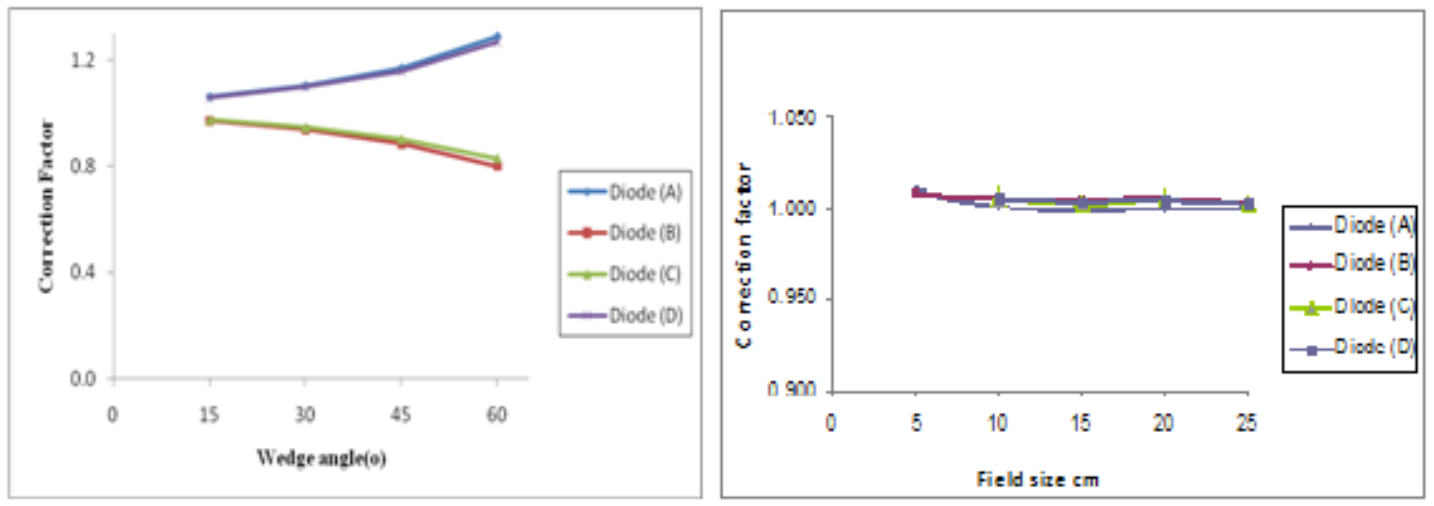

Fig.4. a. shows the effect inserting the wedge in a beam decrease the dose rate and changes the beam quality, (4. b) shows CFs as a function tray plate.

The signal increase with incident angle for all angles from $\left(0^{0}\right.$ to $90^{0},-0^{0}$ to $\left.-90^{\circ}\right)$, the signal was normalized to the standard degree (gantry angle at $0^{\circ}$ ), as shown in fig.5. The signal is about $2.14 \%$ and $2.04 \%$ greater than at $0^{\circ}$ respectively . 


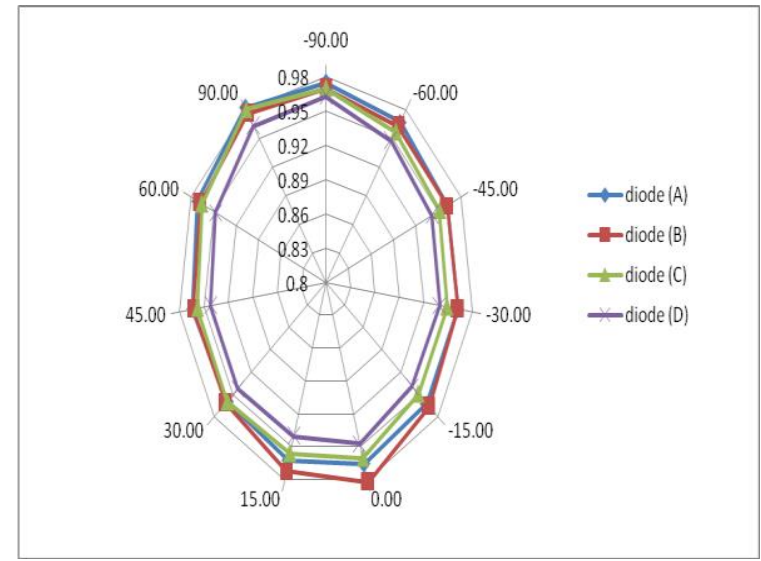

Fig.5. Correction factors as a function og gantry angle.

\section{CONCLUSION}

The aim of this study was to verily the response of diodes $\mathrm{p}$ type (Isorad) use in radiotherapy, to establish calibration procedure for diodes and to evaluate entrance dose obtained by the treatment planning system with measured dose using four Isorad diodes detectors conducts in this study. Our attention focused on entrance dose calibration, as part of quality assurance programme. We have evaluated stability, linearity calibration and correction factors. The results within expected value for this type of diodes giving acceptable agreement in dose delivered and the expected dose. We seek to investigate the effect of other factors on response and sensitivity of the diode, such as exit dose measurements, midline dose calculation, skin dose, critical organ and temperature correction factor. Carry out a patient more studies for different treatment techniques.

\section{RECOMENDATION}

It's recommended to use diode as a dosimeter for monitoring the delivery of dose to patient receiving external beam therapy. In doing so calibration and correction factors should be determined for each diode in all relevant clinical conditions.

\section{ACKOWLEDGEMENT}

We would like to thank Nuclear power engineer Allqe. Ali. I, for his support and help.

\section{REFERENCES}

[1] [1]- Essers M,Mijnheer BJ. In vivo dosimetry during external photon beam radiotherapy. Int J Radiat Oncol Biol Phys 1999;43:245-259.

[2] [2]- Loncol T, Greffe JL, Vynckier S, Scalliet P. Entrance and exit dose measurementwith semiconductors and TLD: a comparison of methods and in vivo results.Radiother Oncol 1996; 41:179-187.

[3] [3]- Jornet N, Ribas M, Eudaldo T. In vivo dosimetry: Intercomparison between p-type and n-type based diodes for the 16-25 MV range. MedPhys 2000; 27:1287-1293

[4] [4]. D. P. Huyskens, R. Bogaerts, J. Verstraete, M. Lööf, H. Nyström, C. Fiorino, S. Broggi, N. Jornet, M. Ribas, D. I. Thwaites, Practical Guidelines for implementation of in-vivo dosimetry with diodes in external radiotherapy with photon beams (entrance dose), ESTRO (2001).

[5] [5]. E. Yorke (Chair), R. Alecu, L. Ding, D. Fontenla, A. Kalend, D. Kaurin, M. E. Masterson-McGary, G. Marinello, T. Matzen, A. Saini, J. Shi, W. Simon, T.C. Zhu, X. R. Zhu, Diode in-vivo dosimetry for patients receiving external beam radiation therapy, AAPM report No 87 Medical Physics Publishing, Madison, (2005).

[6] [6]- Jornt N, Ribas M, Eudaldo T. In vivo dosimetry: Inter-comperation between p-type and n-type based diodes for the 16-25 MV range. Med Phy 2000; 27:1287-129.

[7] [7]- Nilsson B, Ruden B-I, Sorcini B. Characterization of silicon diodes as patient dosemeters in external radiation therapy. Radioother Oncol 1988;11: 279-288.

[8] [8]-Voordeckers M, Goosens H, Rutten J, Van den Bogaert W. The implementation of in vivo dosimetry in a small radiotherapy department. Radiother Oncol 1998; 47:45-48.

[9] [9]- leunens G, Van Dam J, Dutreix A, van der Schueren E. Quality assurance in radiotherapy by in vivo dosimetry. 1. Entrance dose measurements, a reliable procedure. Radiother Oncol 1990; 17: 141-151.

\section{AUTHORS}

First Author - Nureddin. A. S. Musa, Sudan University of Science and Technology, College of Graduate Studies, Scientific Research, P.O. Box 407, Khartoum, Sudan., nureddine2001@yahoo.com

Second Author - Pro. Mohamed Elfadil. M, Sudan University of Science and Technology, College of Graduate Studies, Scientific Research, P.O. Box 407, Khartoum, Sudan, mohamedelfadilmohamed@gmail.com 